Network Working Group

Request for Comments: 2315

Category: Informational

B. Kaliski

RSA Laboratories, East

March 1998

PKCS \#7: Cryptographic Message Syntax

Version 1.5

Status of this Memo

This memo provides information for the Internet community. It does not specify an Internet standard of any kind. Distribution of this memo is unlimited.

Copyright Notice

Copyright (C) The Internet Society (1998). All Rights Reserved.

Overview

This document describes a general syntax for data that may have cryptography applied to it, such as digital signatures and digital envelopes. The syntax admits recursion, so that, for example, one envelope can be nested inside another, or one party can sign some previously enveloped digital data. It also allows arbitrary attributes, such as signing time, to be authenticated along with the content of a message, and provides for other attributes such as countersignatures to be associated with a signature. A degenerate case of the syntax provides a means for disseminating certificates and certificate-revocation lists.

1. Scope

This document is compatible with Privacy-Enhanced Mail (PEM) in that signed-data and signed-and-enveloped-data content, constructed in a PEM-compatible mode, can be converted into PEM messages without any cryptographic operations. PEM messages can similarly be converted into the signed-data and signed-and-enveloped data content types.

This document can support a variety of architectures for certificate-based key management, such as the one proposed for Privacy-Enhanced Mail in RFC 1422. Architectural decisions such as what certificate issuers are considered "top-level," what entities certificate issuers are authorized to certify, what distinguished names are considered acceptable, and what policies certificate issuers must follow (such as signing only with secure hardware, or requiring entities to present specific forms of identification) are left outside the document. 
The values produced according to this document are intended to be BER-encoded, which means that the values would typically be represented as octet strings. While many systems are capable of transmitting arbitrary octet strings reliably, it is well known that many electronic-mail systems are not. This document does not address mechanisms for encoding octet strings as (say) strings of ASCII characters or other techniques for enabling reliable transmission by re-encoding the octet string. RFC 1421 suggests one possible solution to this problem.

2. References

FIPS PUB 46-1 National Bureau of Standards. FIPS PUB 46-1: Data Encryption Standard. January 1988.

PKCS \#1 RSA Laboratories. PKCS \#1: RSA Encryption. Version 1.5, November 1993.

PKCS \#6 RSA Laboratories. PKCS \#6: Extended-Certificate Syntax. Version 1.5, November 1993.

PKCS \#9 RSA Laboratories. PKCS \#9: Selected Attribute Types. Version 1.1, November 1993.

RFC 1421 Linn, J., "Privacy Enhancement for Internet Electronic Mail: Part I: Message Encryption and Authentication Procedures, " RFC 1421 February 1993.

RFC 1422 Kent, S., "Privacy Enhancement for Internet Electronic Mail: Part II: CertificateBased Key Management," RFC 1422, February 1993.

RFC 1423 Balenson, D., "Privacy Enhancement for Internet Electronic Mail: Part III: Algorithms, Modes, and Identifiers," RFC 1423, February 1993.

RFC 1424 Kaliski, B., "Privacy Enhancement for Internet Electronic Mail: Part IV: Key Certification and Related Services," RFC 1424, February 1993. 


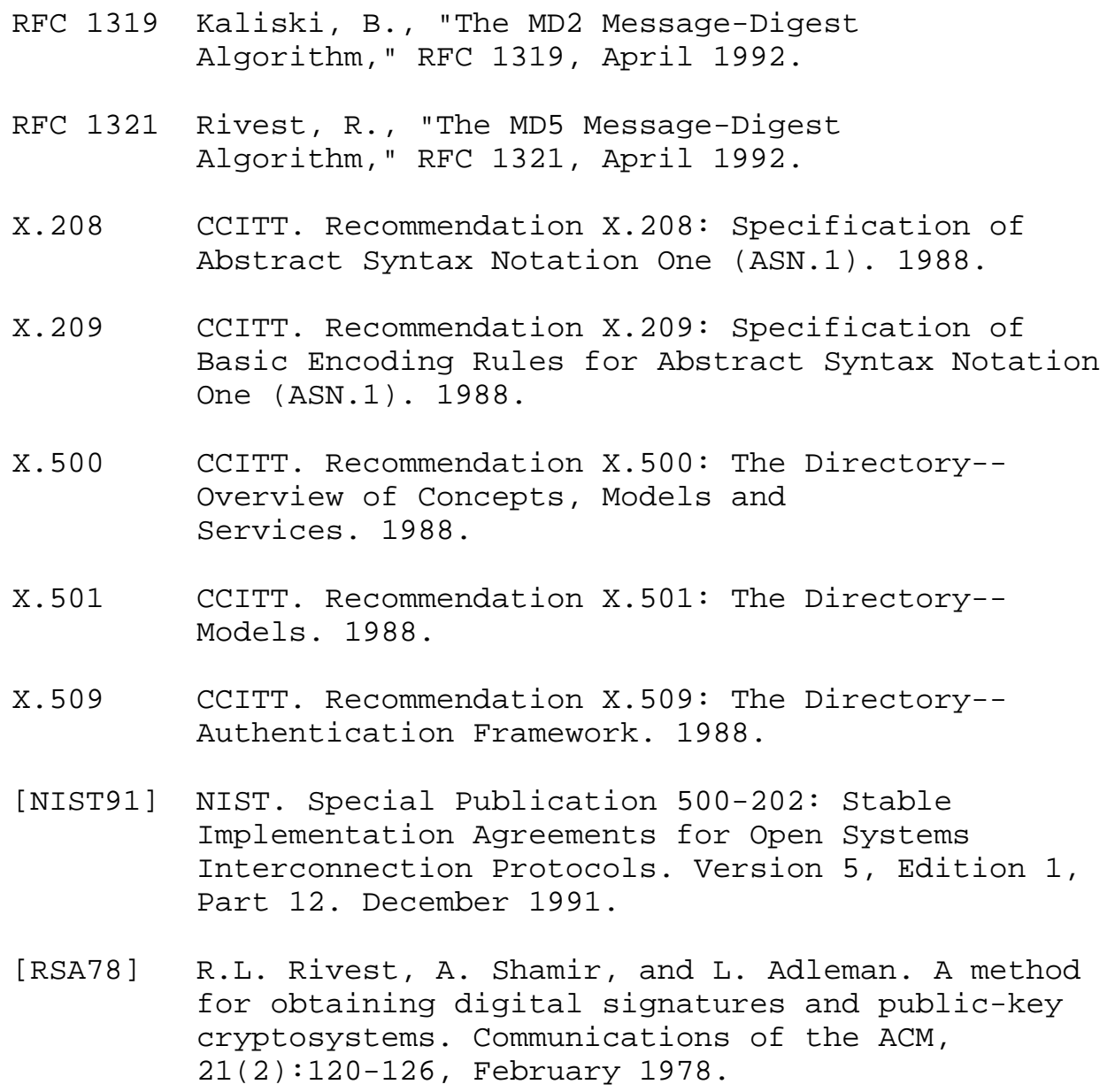

3. Definitions

For the purposes of this document, the following definitions apply. AlgorithmIdentifier: A type that identifies an algorithm (by object identifier) and associated parameters. This type is defined in X.509.

ASN.1: Abstract Syntax Notation One, as defined in X.208.

Attribute: A type that contains an attribute type (specified by object identifier) and one or more attribute values. This type is defined in X.501.

BER: Basic Encoding Rules, as defined in X.209. 
Certificate: A type that binds an entity's distinguished name to a public key with a digital signature. This type is defined in X.509. This type also contains the distinguished name of the certificate issuer (the signer), an issuer-specific serial number, the issuer's signature algorithm identifier, and a validity period.

CertificateserialNumber: A type that uniquely identifies a certificate (and thereby an entity and a public key) among those signed by a particular certificate issuer. This type is defined in $\mathrm{X} .509$.

CertificateRevocationList: A type that contains information about certificates whose validity an issuer has prematurely revoked. The information consists of an issuer name, the time of issue, the next scheduled time of issue, and a list of certificate serial numbers and their associated revocation times. The CRL is signed by the issuer. The type intended by this document is the one defined RFC 1422 .

DER: Distinguished Encoding Rules for ASN.1, as defined in X.509, Section 8.7 .

DES: Data Encryption Standard, as defined in FIPS PUB 46-1. desCBC: The object identifier for DES in cipher-block chaining (CBC) mode, as defined in [NIST91].

ExtendedCertificate: A type that consists of an X.509 public-key certificate and a set of attributes, collectively signed by the issuer of the X.509 public-key certificate. This type is defined in PKCS \# 6 .

MD2: RSA Data Security, Inc.'s MD2 message-digest algorithm, as defined in RFC 1319.

md2: The object identifier for MD2, as defined in RFC 1319.

MD5: RSA Data Security, Inc.'s MD5 message-digest algorithm, as defined in RFC 1321.

md5: The object identifier for MD5, as defined in RFC 1321.

Name: A type that uniquely identifies or "distinguishes" objects in an X.500 directory. This type is defined in X.501. In an X.509 certificate, the type identifies the certificate issuer and the entity whose public key is certified.

PEM: Internet Privacy-Enhanced Mail, as defined in RFCs 1421-1424. 
RSA: The RSA public-key cryptosystem, as defined in [RSA78] . rsaEncryption: The object identifier for RSA encryption, as defined in PKCS \#1.

4. Symbols and abbreviations

No symbols or abbreviations are defined in this document.

5. General overview

The following nine sections specify useful types, general syntax, six content types, and object identifiers.

The syntax is general enough to support many different content types. This document defines six: data, signed data, enveloped data, signed-and-enveloped data, digested data, and encrypted data. Other content types may be added in the future. The use of content types defined outside this document is possible, but is subject to bilateral agreement between parties exchanging content.

This document exports one type, ContentInfo, as well as the various object identifiers.

There are two classes of content types: base and enhanced. Content types in the base class contain "just data," with no cryptographic enhancements. Presently, one content type is in this class, the data content type. Content types in the enhanced class contain content of some type (possibly encrypted), and other cryptographic enhancements. For example, enveloped-data content can contain (encrypted) signeddata content, which can contain data content. The four non-data content types fall into the enhanced class. The content types in the enhanced class thus employ encapsulation, giving rise to the terms "outer" content (the one containing the enhancements) and "inner" content (the one being enhanced).

The document is designed such that the enhanced content types can be prepared in a single pass using indefinite-length BER encoding, and processed in a single pass in any BER encoding. Single-pass operation is especially helpful if content is stored on tapes, or is "piped" from another process. One of the drawbacks of single-pass operation, however, is that it is difficult to output a DER encoding in a single pass, since the lengths of the various components may not be known in advance. Since DER encoding is required by the signed-data, signedand-enveloped data, and digested-data content types, an extra pass may be necessary when a content type other than data is the inner content of one of those content types. 
6. Useful types

This section defines types that are useful in at least two places in the document.

6.1 CertificateRevocationLists

The CertificateRevocationLists type gives a set of certificaterevocation lists. It is intended that the set contain information sufficient to determine whether the certificates with which the set is associated are "hot listed," but there may be more certificaterevocation lists than necessary, or there may be fewer than necessary .

CertificateRevocationLists : := SET OF CertificateRevocationList

\subsection{ContentEncryptionAlgorithmIdentifier}

The ContentEncryptionAlgorithmIdentifier type identifies a contentencryption algorithm such as DES. A content-encryption algorithm supports encryption and decryption operations. The encryption operation maps an octet string (the message) to another octet string (the ciphertext) under control of a content-encryption key. The decryption operation is the inverse of the encryption operation. context determines which operation is intended.

ContentEncryptionAlgorithmIdentifier : :=

AlgorithmIdentifier

6.3 DigestAlgorithmIdentifier

The DigestAlgorithmIdentifier type identifies a message-digest algorithm. Examples include MD2 and MD5. A message-digest algorithm maps an octet string (the message) to another octet string (the message digest).

DigestAlgorithmIdentifier : := AlgorithmIdentifier

\subsection{DigestEncryptionAlgorithmIdentifier}

The DigestEncryptionAlgorithmIdentifier type identifies a digestencryption algorithm under which a message digest can be encrypted. One example is PKCS \#1's rsaEncryption. A digest-encryption algorithm supports encryption and decryption operations. The encryption operation maps an octet string (the message digest) to another octet .bp string (the encrypted message digest) under control of a digestencryption key. The decryption operation is the inverse of the 
encryption operation. Context determines which operation is intended.

DigestEncryptionAlgorithmIdentifier : := AlgorithmIdentifier

6.5 ExtendedCertificateOrCertificate

The ExtendedCertificateOrCertificate type gives either a PKCS \#6 extended certificate or an X.509 certificate. This type follows the syntax recommended in section 6 of PKCS \#6:

ExtendedCertificateOrCertificate : := CHOICE \{

certificate Certificate, -- X.509

extendedCertificate [0] IMPLICIT ExtendedCertificate \}

\section{6 ExtendedCertificatesAndCertificates}

The ExtendedCertificatesAndCertificates type gives a set of extended certificates and X.509 certificates. It is intended that the set be sufficient to contain chains from a recognized "root" or "top-level certification authority" to all of the signers with which the set is associated, but there may be more certificates than necessary, or there may be fewer than necessary.

ExtendedCertificatesAndCertificates : := SET OF ExtendedCertificateOrCertificate

Note. The precise meaning of a "chain" is outside the scope of this document. Some applications of this document may impose upper limits on the length of a chain; others may enforce certain relationships between the subjects and issuers of certificates in a chain. An example of such relationships has been proposed for Privacy-Enhanced Mail in RFC 1422 .

\subsection{IssuerAndSerialNumber}

The IssuerAndSerialNumber type identifies a certificate (and thereby an entity and a public key) by the distinguished name of the certificate issuer and an issuer-specific certificate serial number.

IssuerAndSerialNumber : := SEQUENCE \{ issuer Name, serialNumber CertificateSerialNumber \} 


\subsection{KeyEncryptionAlgorithmIdentifier}

The KeyEncryptionAlgorithmIdentifier type identifies a key-encryption algorithm under which a content-encryption key can be encrypted. One example is PKCS \#1's rsaEncryption. A key-encryption algorithm supports encryption and decryption operations. The encryption operation maps an octet string (the key) to another octet string (the encrypted key) under control of a key-encryption key. The decryption operation is the inverse of the encryption operation. Context determines which operation is intended.

KeyEncryptionAlgorithmIdentifier : := AlgorithmIdentifier

\subsection{Version}

The Version type gives a syntax version number, for compatibility with future revisions of this document.

Version : := INTEGER

7. General syntax

The general syntax for content exchanged between entities according to this document associates a content type with content. The syntax shall have ASN.1 type ContentInfo:

ContentInfo : := SEQUENCE \{ content Type Content Type, content

[0] EXPLICIT ANY DEFINED BY contentType OPTIONAL \}

ContentType : := OBJECT IDENTIFIER

The fields of type ContentInfo have the following meanings:

- content Type indicates the type of content. It is an object identifier, which means it is a unique string of integers assigned by the authority that defines the content type. This document defines six content types (see section 14): data, signedData, envelopedData, signedAndEnvelopedData, digestedData, and encryptedData.

o content is the content. The field is optional, and if the field is not present, its intended value must be supplied by other means. Its type is defined along with the object identifier for contentType. 
Notes

1. The methods below assume that the type of content can be determined uniquely by contentType, so the type defined along with the object identifier should not be a CHOICE type.

2. When a ContentInfo value is the inner content of signed-data, signed-and-enveloped-data, or digested-data content, a message-digest algorithm is applied to the contents octets of the DER encoding of the content field. When a ContentInfo value is the inner content of enveloped-data or signed-and-enveloped-data content, a content-encryption algorithm is applied to the contents octets of a definite-length BER encoding of the content field.

3. The optional omission of the content field makes it possible to construct "external signatures," for example, without modification to or replication of the content to which the signatures apply. In the case of external signatures, the content being signed would be omitted from the "inner" encapsulated ContentInfo value included in the signed-data content type.

8. Data content type

The data content type is just an octet string. It shall have ASN.1 type Data:

Data $::=$ OCTET STRING

The data content type is intended to refer to arbitrary octet strings, such as ASCII text files; the interpretation is left to the application. Such strings need not have any internal structure (although they may; they could even be DER encodings).

9. Signed-data content type

The signed-data content type consists of content of any type and encrypted message digests of the content for zero or more signers. The encrypted digest for a signer is a "digital signature" on the content for that signer. Any type of content can be signed by any number of signers in parallel. Furthermore, the syntax has a degenerate case in which there are no signers on the content. The degenerate case provides a means for disseminating certificates and certificate-revocation lists. 
It is expected that the typical application of the signed-data content type will be to represent one signer's digital signature on content of the data content type. Another typical application will be to disseminate certificates and certificate-revocation lists.

The process by which signed data is constructed involves the following steps:

1. For each signer, a message digest is computed on the content with a signer-specific message-digest algorithm. (If two signers employ the same message-digest algorithm, then the message digest need be computed for only one of them.) If the signer is authenticating any information other than the content (see Section 9.2), the message digest of the content and the other information are digested with the signer's message digest algorithm, and the result becomes the "message digest."

2. For each signer, the message digest and associated information are encrypted with the signer's private key.

3. For each signer, the encrypted message digest and other signer-specific information are collected into a SignerInfo value, defined in section 9.2. Certificates and certificate-revocation lists for each signer, and those not corresponding to any signer, are collected in this step.

4. The message-digest algorithms for all the signers and the SignerInfo values for all the signers are collected together with the content into a SignedData value, defined in section 9.1 .

A recipient verifies the signatures by decrypting the encrypted message digest for each signer with the signer's public key, then comparing the recovered message digest to an independently computed message digest. The signer's public key is either contained in a certificate included in the signer information, or is referenced by an issuer distinguished name and an issuer-specific serial number that uniquely identify the certificate for the public key.

This section is divided into five parts. The first part describes the top-level type SignedData, the second part describes the per-signer information type SignerInfo, and the third and fourth parts describe the message-digesting and digest-encryption processes. The fifth part summarizes compatibility with Privacy-Enhanced Mail. 


\subsection{SignedData type}

The signed-data content type shall have ASN.1 type SignedData:

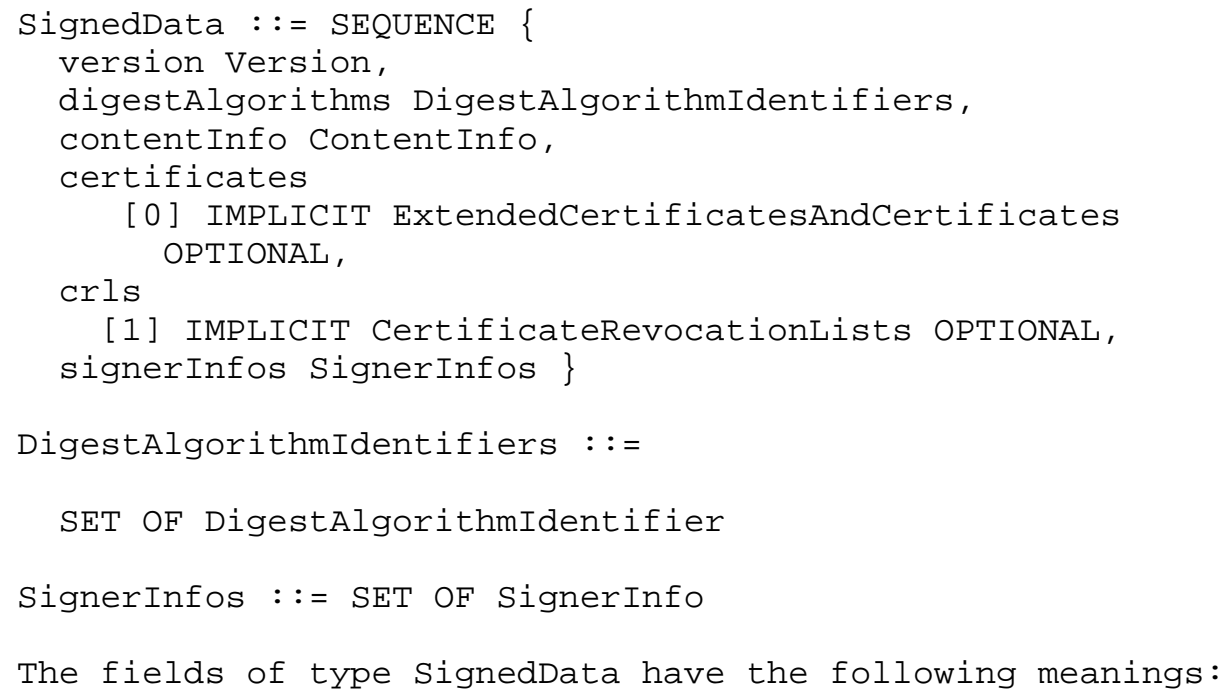

[1] IMPLICIT CertificateRevocationLists OPTIONAL, signerInfos SignerInfos \}

DigestAlgorithmIdentifiers : :=

SET OF DigestAlgorithmIdentifier

SignerInfos : := SET OF SignerInfo

The fields of type SignedData have the following meanings:

o version is the syntax version number. It shall be 1 for this version of the document.

o digestAlgorithms is a collection of message-digest algorithm identifiers. There may be any number of elements in the collection, including zero. Each element identifies the message-digest algorithm (and any associated parameters) under which the content is digested for a some signer. The collection is intended to list the message-digest algorithms employed by all of the signers, in any order, to facilitate one-pass signature verification. The message-digesting process is described in section 9.3.

o contentInfo is the content that is signed. It can have any of the defined content types.

- certificates is a set of PKCS \#6 extended certificates and X.509 certificates. It is intended that the set be sufficient to contain chains from a recognized "root" or "top-level certification authority" to all of the signers in the signerInfos field. There may be more certificates than necessary, and there may be certificates sufficient to contain chains from two or more independent 
top-level certification authorities. There may also be fewer certificates than necessary, if it is expected that those verifying the signatures have an alternate means of obtaining necessary certificates (e.g., from a previous set of certificates).

crls is a set of certificate-revocation lists. It is intended that the set contain information sufficient to determine whether or not the certificates in the certificates field are "hot listed," but such correspondence is not necessary. There may be more certificate-revocation lists than necessary, and there may also be fewer certificate-revocation lists than necessary.

- signerInfos is a collection of per-signer information. There may be any number of elements in the collection, including zero.

Notes.

1. The fact that the digestAlgorithms field comes before the contentInfo field and the signerInfos field comes after it makes it possible to process a signedData value in a single pass. (Single-pass processing is described in section 5.)

2. The differences between version 1 SignedData and version 0 SignedData (defined in PKCS \#7, Version 1.4) are the following:

the digestAlgorithms and signerInfos fields may contain zero elements in version 1 , but not in version 0

$\circ$ the crls field is allowed in version 1 , but not in version 0

Except for the difference in version number, version 0 SignedData values are acceptable as version 1 values. An implementation can therefore process SignedData values of either version as though they were version 1 values. It is suggested that PKCS implementations generate only version 1 SignedData values, but be prepared to process SignedData values of either version. 
3. In the degenerate case where there are no signers on the content, the contentInfo value being "signed" is irrelevant. It is recommended in that case that the content type of the ContentInfo value being "signed" be data, and the content field of the contentInfo value be omitted.

\subsection{SignerInfo type}

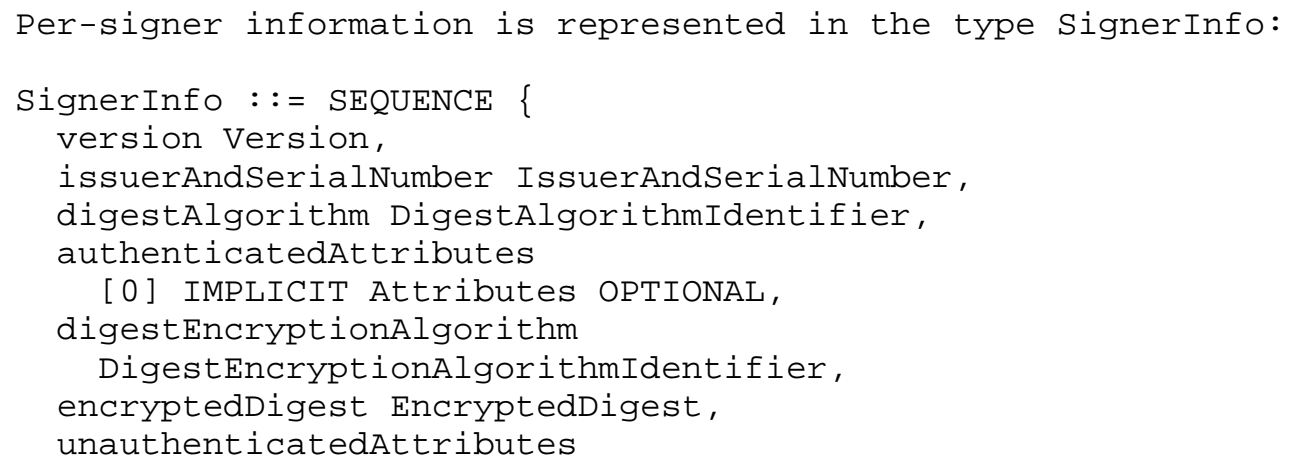

- digestAlgorithm identifies the message-digest algorithm (and any associated parameters) under which the content and authenticated attributes (if present) are digested. It should be among those in the digestAlgorithms field of the superior SignerInfo value. The messagedigesting process is described in section 9.3.

o authenticatedAttributes is a set of attributes that are signed (i.e., authenticated) by the signer. The field is optional, but it must be present if the content type of the ContentInfo value being signed is not data. If the field is present, it must contain, at a minimum, two attributes: 
1. A PKCS \#9 content-type attribute having as its value the content type of the ContentInfo value being signed.

2. A PKCS \#9 message-digest attribute, having as its value the message digest of the content (see below).

Other attribute types that might be useful here, such as signing time, are also defined in PKCS \#9.

$\bigcirc$

O

○

It is recommended in the interest of PEM compatibility that the authenticatedAttributes field be omitted whenever the content type of the ContentInfo value being signed is data and there are no other authenticated attributes.

2. The difference between version 1 SignerInfo and version 0 SignerInfo (defined in PKCS \#7, Version 1.4) is in the message-digest encryption process (see Section 9.4). Only the PEM-compatible processes are different, reflecting changes in Privacy-Enhanced Mail signature methods. There is no difference in the non-PEM-compatible message-digest encryption process.

It is suggested that PKCS implementations generate only version 1 SignedData values. Since the PEM signature method with which version 0 is compatible is obsolescent, it is suggested that PKCS implementations be prepared to receive only version 1 SignedData values. 


\subsection{Message-digesting process}

The message-digesting process computes a message digest on either the content being signed or the content together with the signer's authenticated attributes. In either case, the initial input to the message-digesting process is the "value" of the content being signed. Specifically, the initial input is the contents octets of the DER encoding of the content field of the ContentInfo value to which the signing process is applied. Only the contents octets of the DER encoding of that field are digested, not the identifier octets or the length octets.

The result of the message-digesting process (which is called, informally, the "message digest") depends on whether the authenticatedAttributes field is present. When the field is absent, the result is just the message digest of the content. When the field is present, however, the result is the message digest of the complete DER encoding of the Attributes value containted in the authenticatedAttributes field. (For clarity: The IMPLICIT [0] tag in the authenticatedAttributes field is not part of the Attributes value. The Attributes value's tag is SET OF, and the DER encoding of the SET OF tag, rather than of the IMPLICIT [0] tag, is to be digested along with the length and contents octets of the Attributes value.) Since the Attributes value, when the field is present, must contain as attributes the content type and the message digest of the content, those values are indirectly included in the result.

When the content being signed has content type data and the authenticatedAttributes field is absent, then just the value of the data (e.g., the contents of a file) is digested. This has the advantage that the length of the content being signed need not be known in advance of the encryption process. This method is compatible with Privacy-Enhanced Mail.

Although the identifier octets and the length octets are not digested, they are still protected by other means. The length octets are protected by the nature of the message-digest algorithm since it is by assumption computationally infeasible to find any two distinct messages of any length that have the same message digest. Furthermore, assuming that the content type uniquely determines the identifier octets, the identifier octets are protected implicitly in one of two ways: either by the inclusion of the content type in the authenticated attributes, or by the use of the PEM-compatible alternative in section 9.4 which implies that the content type is data. 
Note. The fact that the message digest is computed on part of a DER encoding does not mean that DER is the required method of representing that part for data transfer. Indeed, it is expected that some implementations of this document may store objects in other than their DER encodings, but such practices do not affect message-digest computation.

\subsection{Digest-encryption process}

The input to the digest-encryption process--the value supplied to the signer's digest-encryption algorithm--includes the result of the message-digesting process (informally, the "message digest") and the digest algorithm identifier (or object identifier). The result of the digest-encryption process is the encryption with the signer's private key of the BER encoding of a value of type DigestInfo:

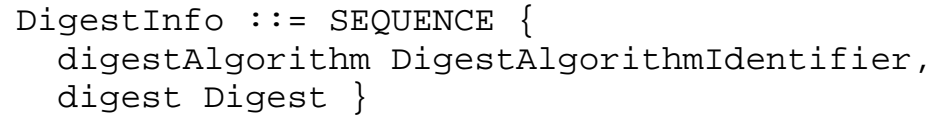

o digestAlgorithm identifies the message-digest algorithm (and any associated parameters) under which the content and authenticated attributes are digested. It should be the same as the digestAlgorithm field of the superior SignerInfo value.

o digest is the result of the message-digesting process.

Notes.

1. The only difference between the signature process defined here and the signature algorithms defined in PKCS \#1 is that signatures there are represented as bit strings, for consistency with the X.509 SIGNED macro. Here, encrypted message digests are octet strings.

2. The input to the encryption process typically will have 30 or fewer octets. If digestEncryptionAlgorithm is PKCS \#1's rsaEncryption, then this means that the input can be encrypted in a single block as long as the length of the RSA modulus is at least 328 bits, which is reasonable and consistent with security recommendations. 
3. A message-digest algorithm identifier is included in the DigestInfo value to limit the damage resulting from the compromise of one message-digest algorithm. For instance, suppose an adversary were able to find messages with a given MD2 message digest. That adversary could then forge a signature by finding a message with the same MD2 message digest as one that a signer previously signed, and presenting the previous signature as the signature on the new message. This attack would succeed only if the signer previously used MD2, since the DigestInfo value contains the message-digest algorithm. If a signer never trusted the MD2 algorithm and always used MD5, then the compromise of MD2 would not affect the signer. If the DigestInfo value contained only the message digest, however, the compromise of MD2 would affect signers that use any message-digest algorithm.

4. There is potential for ambiguity due to the fact that the DigestInfo value does not indicate whether the digest field contains just the message digest of the content or the message digest of the complete DER encoding of the authenticatedAttributes field. In other words, it is possible for an adversary to transform a signature on authenticated attributes to one that appears to be just on content by changing the content to be the DER encoding of the authenticatedAttributes field, and then removing the authenticatedAttributes field. (The reverse transformation is possible, but requires that the content be the DER encoding of an authenticated attributes value, which is unlikely.) This ambiguity is not a new problem, nor is it a significant one, as context will generally prevent misuse. Indeed, it is also possible for an adversary to transform a signature on a certificate or certificate-revocation list to one that appears to be just on signed-data content.

\subsection{Compatibility with Privacy-Enhanced Mail}

Compatibility with the MIC-ONLY and MIC-CLEAR process types in PEM occurs when the content type of the ContentInfo value being signed is data, there are no authenticated attributes, the message-digest algorithm is md2 or md5, and the digest-encryption algorithm is PKCS \#1's rsaEncryption. Under all those conditions, the encrypted message digest produced here matches the one produced in PEM because:

1. The value input to the message-digest algorithm in PEM is the same as in this document when there are no authenticated attributes. MD2 and MD5 in PEM are the same as md2 and md5. 
2. The value encrypted with the signer's private key in PEM (as specified in RFC 1423) is the same as in this document when there are no authenticated attributes. RSA private-key encryption in PEM is the same as PKCS \#1's rsaEncryption.

The other parts of the signed-data content type (certificates, CRLs, algorithm identifiers, etc.) are easily translated to and from their corresponding PEM components.

10. Enveloped-data content type

The enveloped-data content type consists of encrypted content of any type and encrypted content-encryption keys for one or more recipients. The combination of encrypted content and encrypted content-encryption key for a recipient is a "digital envelope" for that recipient. Any type of content can be enveloped for any number of recipients in parallel.

It is expected that the typical application of the enveloped-data content type will be to represent one or more recipients' digital envelopes on content of the data, digested-data, or signed-data content types.

The process by which enveloped data is constructed involves the following steps:

1. A content-encryption key for a particular contentencryption algorithm is generated at random.

2. For each recipient, the content-encryption key is encrypted with the recipient's public key.

3. For each recipient, the encrypted contentencryption key and other recipient-specific information are collected into a RecipientInfo value, defined in Section 10.2 .

4. The content is encrypted with the contentencryption key. (Content encryption may require that the content be padded to a multiple of some block size; see Section 10.3 for discussion.)

5. The RecipientInfo values for all the recipients are collected together with the encrypted content into a EnvelopedData value, defined in section 10.1. 
A recipient opens the envelope by decrypting the one of the encrypted content-encryption keys with the recipient's private key and decrypting the encrypted content with the recovered contentencryption key. The recipient's private key is referenced by an issuer distinguished name and an issuer-specific serial number that uniquely identify the certificate for the corresponding public key.

This section is divided into four parts. The first part describes the top-level type EnvelopedData, the second part describes the perrecipient information type RecipientInfo, and the third and fourth parts describe the content-encryption and key-encryption processes.

This content type is not compatible with Privacy-Enhanced Mail (although some processes it defines are compatible with their PEM counterparts), since Privacy-Enhanced Mail always involves digital signatures, never digital envelopes alone.

\subsection{EnvelopedData type}

The enveloped-data content type shall have ASN.1 type EnvelopedData:

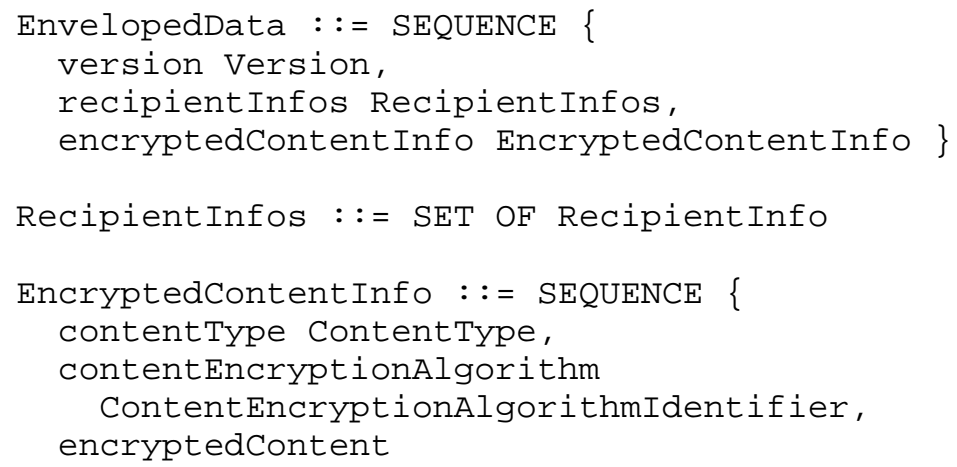

[0] IMPLICIT EncryptedContent OPTIONAL \}

EncryptedContent : := OCTET STRING

The fields of type EnvelopedData have the following meanings:

- version is the syntax version number. It shall be 0 for this version of the document.

o recipientInfos is a collection of per-recipient information. There must be at least one element in the collection.

- encryptedContentinfo is the encrypted content information. 
The fields of type EncryptedContentInfo have the following meanings:

o content Type indicates the type of content.

- contentEncryptionAlgorithm identifies the contentencryption algorithm (and any associated parameters) under which the content is encrypted. The content-encryption process is described in Section 10.3. This algorithm is the same for all recipients.

o encryptedContent is the result of encrypting the content. The field is optional, and if the field is not present, its intended value must be supplied by other means.

Note. The fact that the recipientInfos field comes before the encryptedContentInfo field makes it possible to process an EnvelopedData value in a single pass. (Single-pass processing is described in section 5.)

\subsection{RecipientInfo type}

Per-recipient information is represented in the type RecipientInfo:

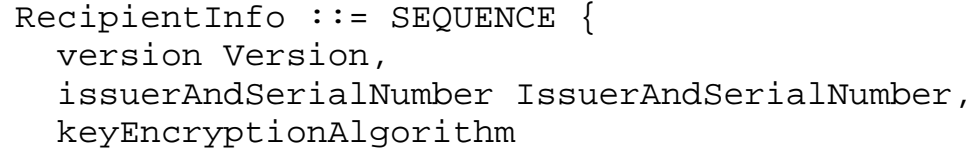

The fields of type RecipientInfo have the following meanings:

o version is the syntax version number. It shall be 0 for this version of the document.

- issuerAndSerialNumber specifies the recipient's certificate (and thereby the recipient's distinguished name and public key) by issuer distinguished name and issuer-specific serial number. 
keyEncryptionAlgorithm identifies the keyencryption algorithm (and any associated parameters) under which the content-encryption key is encrypted with the recipient's public key. The key-encryption process is described in section 10.4 .

- encryptedkey is the result of encrypting the content-encryption key with the recipient's public key (see below).

\subsection{Content-encryption process}

The input to the content-encryption process is the "value" of the content being enveloped. Specifically, the input is the contents octets of a definite-length BER encoding of the content field of the Contentinfo value to which the enveloping process is applied. Only the contents octets of the BER encoding are encrypted, not the identifier octets or length octets; those other octets are not represented at all.

When the content being enveloped has content type data, then just the value of the data (e.g., the contents of a file) is encrypted. This has the advantage that the length of the content being encrypted need not be known in advance of the encryption process. This method is compatible with Privacy-Enhanced Mail.

The identifier octets and the length octets are not encrypted. The length octets may be protected implicitly by the encryption process, depending on the encryption algorithm. The identifier octets are not protected at all, although they can be recovered from the content type, assuming that the content type uniquely determines the identifier octets. Explicit protection of the identifier and length octets requires that the signed-and-enveloped-data content type be employed, or that the digested-data and enveloped-data content types be applied in succession.

Notes.

1. The reason that a definite-length BER encoding is required is that the bit indicating whether the length is definite or indefinite is not recorded anywhere in the enveloped-data content type. Definite-length encoding is more appropriate for simple types such as octet strings, so definite-length encoding is chosen. 
2. Some content-encryption algorithms assume the input length is a multiple of $\mathrm{k}$ octets, where $\mathrm{k}>1$, and let the application define a method for handling inputs whose lengths are not a multiple of $\mathrm{k}$ octets. For such algorithms, the method shall be to pad the input at the trailing end with $\mathrm{k}$ - $(1$ mod $\mathrm{k})$ octets all having value k $(1 \bmod k)$, where $l$ is the length of the input. In other words, the input is padded at the trailing end with one of the following strings:

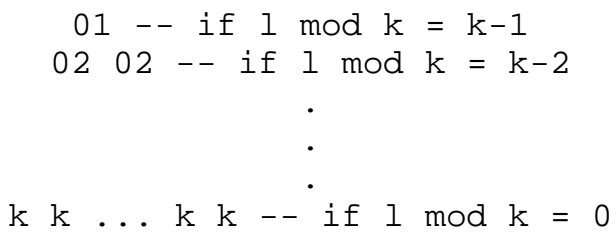

The padding can be removed unambiguously since all input is padded and no padding string is a suffix of another. This padding method is well-defined if and only if $k<256$; methods for larger $k$ are an open issue for further study.

\subsection{Key-encryption process}

The input to the key-encryption process--the value supplied to the recipient's key-encryption algorithm--is just the "value" of the content-encryption key.

11. Signed-and-enveloped-data content type

This section defines the signed-and-enveloped-data content type. For brevity, much of this section is expressed in terms of material in Sections 9 and 10 .

The signed-and-enveloped-data content type consists of encrypted content of any type, encrypted content-encryption keys for one or more recipients, and doubly encrypted message digests for one or more signers. The "double encryption" consists of an encryption with a signer's private key followed by an encryption with the contentencryption key.

The combination of encrypted content and encrypted content-encryption key for a recipient is a "digital envelope" for that recipient. The recovered singly encrypted message digest for a signer is a "digital signature" on the recovered content for that signer. Any type of content can be enveloped for any number of recipients and signed by any number of signers in parallel. 
It is expected that the typical application of the signed-andenveloped-data content type will be to represent one signer's digital signature and one or more recipients' digital envelopes on content of the data content type.

The process by which signed-and-enveloped data is constructed involves the following steps:

1. A content-encryption key for a particular contentencryption algorithm is generated at random.

2. For each recipient, the content-encryption key is encrypted with the recipient's public key.

3. For each recipient, the encrypted contentencryption key and other recipient-specific information are collected into a RecipientInfo value, defined in section 10.2.

4. For each signer, a message digest is computed on the content with a signer-specific message-digest algorithm. (If two signers employ the same messagedigest algorithm, then the message digest need be computed for only one of them.)

5. For each signer, the message digest and associated information are encrypted with the signer's private key, and the result is encrypted with the content-encryption key. (The second encryption may require that the result of the first encryption be padded to a multiple of some block size; see Section 10.3 for discussion.)

6. For each signer, the doubly encrypted message digest and other signer-specific information are collected into a SignerInfo value, defined in Section 9.2 .

7. The content is encrypted with the contentencryption key. (See Section 10.3 for discussion.)

8. The message-digest algorithms for all the signers, the SignerInfo values for all the signers and the RecipientInfo values for all the recipients are collected together with the encrypted content into a SignedAndEnvelopedData value, defined in section 11.1 . 
A recipient opens the envelope and verifies the signatures in two steps. First, the one of the encrypted content-encryption keys is decrypted with the recipient's private key, and the encrypted content is decrypted with the recovered content-encryption key. Second, the doubly encrypted message digest for each signer is decrypted with the recovered content-encryption key, the result is decrypted with the signer's public key, and the recovered message digest is compared to an independently computed message digest.

Recipient private keys and signer public keys are contained or referenced as discussed in Sections 9 and 10.

This section is divided into three parts. The first part describes the top-level type SignedAndEnvelopedData and the second part describes the digest-encryption process. Other types and processes are the same as in sections 9 and 10. The third part summarizes compatibility with Privacy-Enhanced Mail.

Note. The signed-and-enveloped-data content type provides cryptographic enhancements similar to those resulting from the sequential combination of signed-data and enveloped-data content types. However, since the signed-and-enveloped-data content type does not have authenticated or unauthenticated attributes, nor does it provide enveloping of signer information other than the signature, the sequential combination of signed-data and enveloped-data content types is generally preferable to the SignedAndEnvelopedData content type, except when compatibility with the ENCRYPTED process type in Privacy-Enhanced Mail in intended.

\subsection{SignedAndEnvelopedData type}

The signed-and-enveloped-data content type shall have ASN.1 type SignedAndEnvelopedData:

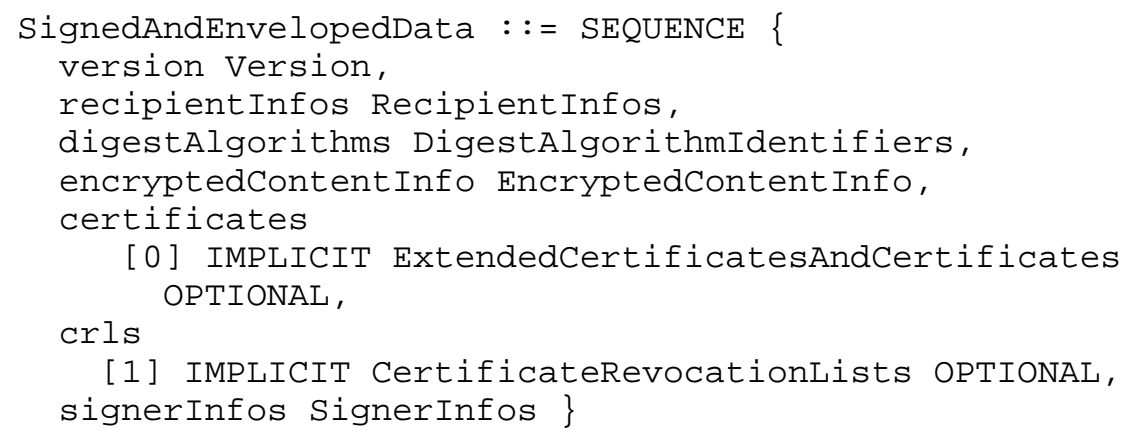


The fields of type SignedAndEnvelopedData have the following meanings :

o version is the syntax version number. It shall be 1 for this version of the document.

- recipientInfos is a collection of per-recipient information, as in section 10 . There must be at least one element in the collection.

- digestAlgorithms is a collection of message-digest algorithm identifiers, as in section 9. The message-digesting process is the same as in Section 9 in the case when there are no authenticated attributes.

- encryptedContentInfo is the encrypted content, as in Section 10. It can have any of the defined content types.

$\circ$ certificates is a set of PKCS \#6 extended certificates and X.509 certificates, as in section 9 .

- signerinfos is a collection of per-signer information. There must be at least one element in the collection. SignerInfo values have the same meaning as in section 9 with the exception of the encryptedDigest field (see below).

Notes.

1. The fact that the recipientInfos and digestAlgorithms fields come before the contentInfo field and the signerInfos field comes after it makes it possible to process a SignedAndEnvelopedData value in a single pass. (Single-pass processing is described in section 5.)

2. The difference between version 1

SignedAndEnvelopedData and version 0 SignedAndEnvelopedData (defined in PKCS \#7, Version 1.4) is that the crls field is allowed in version 1 , but not in version 0 . Except for the difference in version number, version 0 SignedAndEnvelopedData values are acceptable as version 1 values. An implementation can therefore process 
SignedAndEnvelopedData values of either version as though they were version 1 values. It is suggested that PKCS implementations generate only version 1 SignedAndEnvelopedData values, but be prepared to process SignedAndEnvelopedData values of either version.

\subsection{Digest-encryption process}

The input to the digest-encryption process is the same as in Section 9, but the process itself is different. Specifically, the process involves two steps. First, the input to the process is supplied to the signer's digest-encryption algorithm, as in Section 9. Second, the result of the first step is encrypted with the content-encryption key. There is no DER encoding between the two steps; the "value" output by the first step is input directly to the second step. (See Section 10.3 for discussion.)

This process is compatible with the ENCRYPTED process type in Privacy-Enhanced Mail.

Note. The purpose of the second step is to prevent an adversary from recovering the message digest of the content. Otherwise, an adversary would be able to determine which of a list of candidate contents (e.g., "Yes" or "No") is the actual content by comparing the their message digests to the actual message digest.

\subsection{Compatibility with Privacy-Enhanced Mail}

Compatibility with the ENCRYPTED process type of PEM occurs when the content type of the contentInfo value being signed and enveloped is data, the message-digest algorithm is md2 or md5, the contentencryption algorithm is DES in CBC mode, the digest-encryption algorithm is PKCS \#1's rsaEncryption, and the key-encryption algorithm is PKCS \#1's rsaEncryption. Under all those conditions, the doubly encrypted message digest and the encrypted content encryption key match the ones produced in PEM because of reasons similar to those given in Section 9.5, as well as the following:

1. The value input to the content-encryption algorithm in PEM is the same as in this document. DES in CBC mode is the same as desCBC.

2. The value input to the key-encryption algorithm in PEM is the same as in this document (see section 10.4). RSA public-key encryption in PEM is the same as PKCS \#1's rsaEncryption. 
3. The double-encryption process applied to the message digest in this document and in PEM are the same.

The other parts of the signed-and-enveloped-data content type (certificates, CRLs, algorithm identifiers, etc.) are easily translated to and from their corresponding PEM components. (CRLs are carried in a separate PEM message.)

12. Digested-data content type

The digested-data content type consists of content of any type and a message digest of the content.

It is expected that the typical application of the digested-data content type will be to add integrity to content of the data content type, and that the result would become the content input to the enveloped-data content type.

The process by which digested-data is constructed involves the following steps:

1. A message digest is computed on the content with a message-digest algorithm.

2. The message-digest algorithm and the message digest are collected together with the content into a DigestedData value.

A recipient verifies the message digest by comparing the message digest to an independently computed message digest.

The digested-data content type shall have ASN.1 type DigestedData:

DigestedData : := SEQUENCE \{

version Version, digestAlgorithm DigestAlgorithmIdentifier, contentInfo ContentInfo, digest Digest \}

Digest $::=$ OCTET STRING

The fields of type DigestedData have the following meanings:

o version is the syntax version number. It shall be 0 for this version of the document. 
$\bigcirc$

digestAlgorithm identifies the message-digest algorithm (and any associated parameters) under which the content is digested. (The message-digesting process is the same as in section 9 in the case when there are no authenticated attributes.)

- contentInfo is the content that is digested. It can have any of the defined content types.

o digest is the result of the message-digesting process.

Note. The fact that the digestAlgorithm field comes before the contentInfo field and the digest field comes after it makes it possible to process a DigestedData value in a single pass. (Singlepass processing is described in section 5.)

13. Encrypted-data content type

The encrypted-data content type consists of encrypted content of any type. Unlike the enveloped-data content type, the encrypted-data content type has neither recipients nor encrypted content-encryption keys. Keys are assumed to be managed by other means.

It is expected that the typical application of the encrypted-data content type will be to encrypt content of the data content type for local storage, perhaps where the encryption key is a password.

The encrypted-data content type shall have ASN.1 type EncryptedData:

EncryptedData : := SEQUENCE \{

version Version, encryptedContentInfo EncryptedContentInfo \}

The fields of type EncryptedData have the following meanings:

- version is the syntax version number. It shall be 0 for this version of the document.

o encryptedContentInfo is the encrypted content information, as in section 10 .

14. Object identifiers

This document defines seven object identifiers: pkcs-7, data, signedData, envelopedData, signedAndEnvelopedData, digestedData, and encryptedData. 
The object identifier pkcs-7 identifies this document.

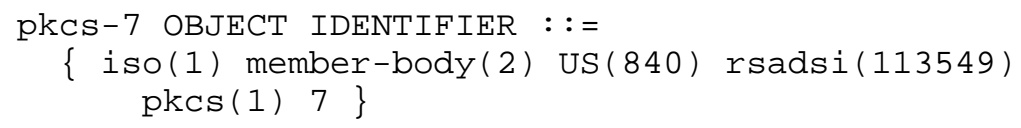




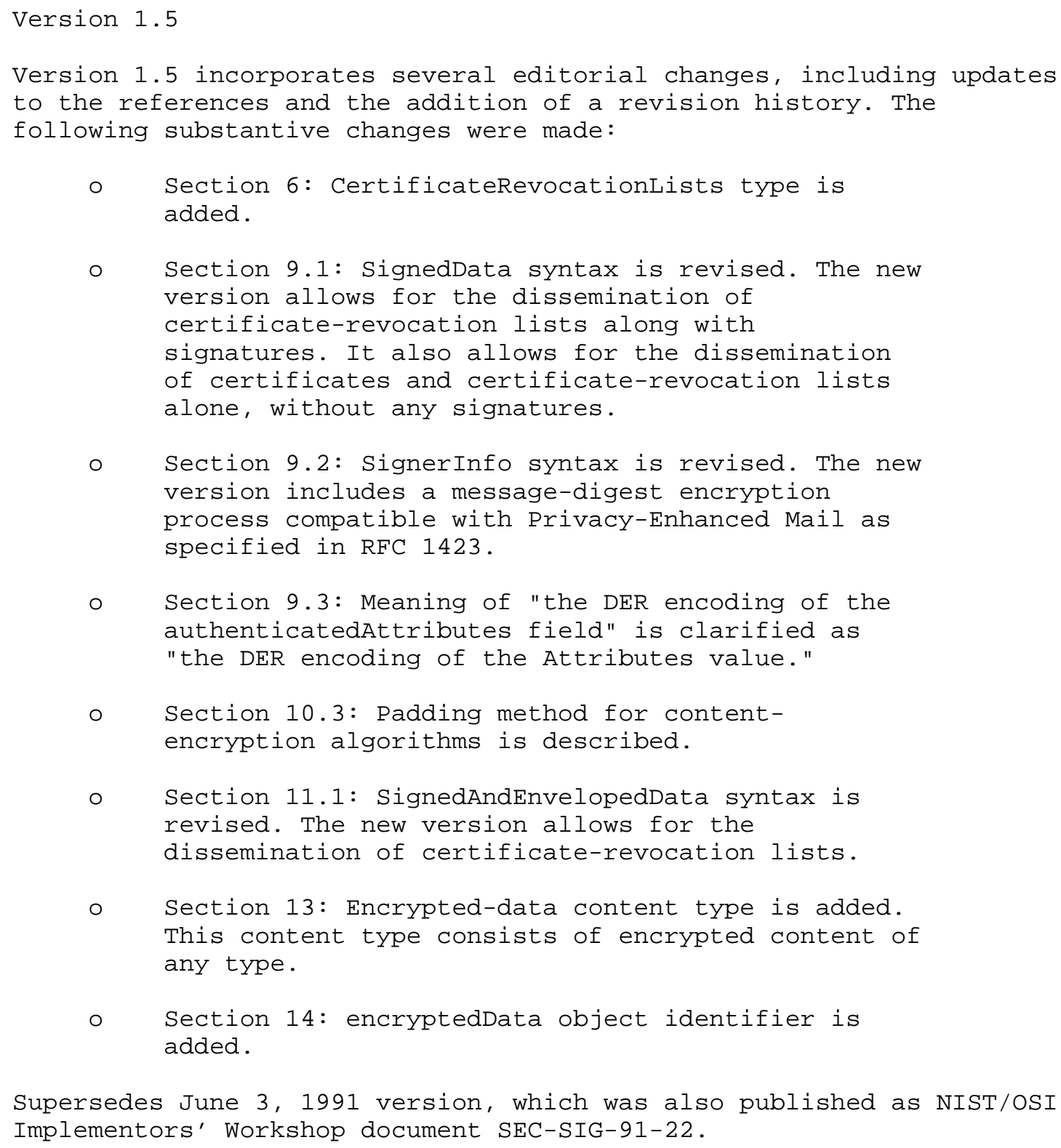


Acknowledgements

This document is based on a contribution of RSA Laboratories, a division of RSA Data Security, Inc. Any substantial use of the text from this document must acknowledge RSA Data Security, Inc. RSA Data Security, Inc. requests that all material mentioning or referencing this document identify this as "RSA Data security, Inc. PKCS \#7".

Author's Address

Burt Kaliski

RSA Laboratories East

20 Crosby Drive

Bedford, MA 01730

Phone: (617) 687-7000

EMail: burtersa.com 
Full Copyright statement

Copyright (C) The Internet Society (1998). All Rights Reserved.

This document and translations of it may be copied and furnished to others, and derivative works that comment on or otherwise explain it or assist in its implementation may be prepared, copied, published and distributed, in whole or in part, without restriction of any kind, provided that the above copyright notice and this paragraph are included on all such copies and derivative works. However, this document itself may not be modified in any way, such as by removing the copyright notice or references to the Internet society or other Internet organizations, except as needed for the purpose of developing Internet standards in which case the procedures for copyrights defined in the Internet Standards process must be followed, or as required to translate it into languages other than English.

The limited permissions granted above are perpetual and will not be revoked by the Internet society or its successors or assigns.

This document and the information contained herein is provided on an "AS IS" basis and THE INTERNET SOCIETY AND THE INTERNET ENGINEERING TASK FORCE DISCLAIMS ALL WARRANTIES, EXPRESS OR IMPLIED, INCLUDING BUT NOT LIMITED TO ANY WARRANTY THAT THE USE OF THE INFORMATION HEREIN WILL NOT INFRINGE ANY RIGHTS OR ANY IMPLIED WARRANTIES OF MERCHANTABILITY OR FITNESS FOR A PARTICULAR PURPOSE. 